\title{
From magnetic order to quantum disorder in the Zn-barlowite series of $S=1 / 2$ kagomé antiferromagnets
}

\author{
Katherine Tustain (1D) ${ }^{1}$, Brendan Ward-O'Brien (iD) ${ }^{1}$, Fabrice Bert $\mathbb{D}^{2}$, Tianheng Han ${ }^{3,4}$, Hubertus Luetkens ${ }^{5}$, Tom Lancaster $\mathbb{D}^{6}$, \\ Benjamin M. Huddart (iD ${ }^{6}$, Peter J. Baker (iD) and Lucy Clark (D) $^{1,8 凶}$
}

We report a comprehensive muon spectroscopy study of the $\mathrm{Zn}$-barlowite series of $S=\frac{1}{2}$ kagomé antiferromagnets, $\mathrm{Zn}{ }_{x} \mathrm{Cu} 4-x(\mathrm{OH})_{6} \mathrm{FBr}$, for $x=0.00$ to $0.99(1)$. By combining muon spin relaxation and rotation measurements with state-of-the-art density-functional theory muon-site calculations, we observe the formation of both $\mu-\mathrm{F}$ and $\mu-\mathrm{OH}$ complexes in $\mathrm{Zn}$-barlowite. From these stopping sites, implanted muon spins reveal the suppression of long-range magnetic order into a possible quantum spin liquid state upon the increasing concentration of Zn-substitution. In the parent compound $(x=0)$, static long-range magnetic order below $T_{\mathrm{N}}=15 \mathrm{~K}$ manifests itself in the form of spontaneous oscillations in the time-dependent muon asymmetry signal consistent with the dipolar fields expected from the calculated muon stopping sites and the previously determined magnetic structure of barlowite. Meanwhile, in the $x=1.0$ end-member of the series-in which antiferromagnetic kagomé layers of $\mathrm{Cu}^{2+} \mathrm{S}=\frac{1}{2}$ moments are decoupled by diamagnetic $\mathrm{Zn}^{2+}$ ions-we observe that dynamic magnetic moment fluctuations persist down to at least $50 \mathrm{mK}$, indicative of a quantum disordered ground state. We demonstrate that this crossover from a static to dynamic magnetic ground state occurs for compositions of Zn-barlowite with $x>0.5$, which bears resemblance to the dynamical behaviour of the widely studied Zn-paratacamite series that contains the quantum spin liquid candidate herbertsmithite.

npj Quantum Materials (2020)5:74; https://doi.org/10.1038/s41535-020-00276-4

\section{INTRODUCTION}

Quantum effects play a significant role in the low-temperature physics of magnetic systems in which antiferromagnetic $S=\frac{1}{2}$ moments decorate a two-dimensional kagomé array of cornersharing triangles ${ }^{1}$. The competing interactions resulting from the geometric frustration in such systems combined with quantum fluctuations may give rise to elusive states of matter, such as quantum spin liquids (QSLs); a possibility that continues to capture the interest of the quantum materials research community $^{2}$. Indeed, QSLs represent a unique state of matter that evades long-range magnetic order despite the sometimes considerable exchange interactions between its magnetic moments, which would typically drive a symmetry-breaking magnetic phase transition in a conventional magnet following Landau theory ${ }^{3}$. Instead, QSLs are quantum superpositional states formed from entangled pairs of magnetic moments, or spins, that can, in certain cases, be envisioned within a resonating valence bond model $^{4}$. QSLs are of fundamental importance as their highly entangled nature should give rise to exotic physical phenomena, such as topological phases, fractionalised excitations and emergent gauge fields ${ }^{5}$. However, the unambiguous realisation of a QSL remains an outstanding challenge.

In particular, while from a theoretical perspective it is generally accepted that the ground state of the $S=\frac{1}{2}$ kagomé antiferromagnet is a $\mathrm{QSL}^{6}$, the true nature of this putative QSL state is still under debate, and new material realisations of it are needed to help resolve the ongoing disconnect between theory and experiment ${ }^{7-9}$. In this regard, synthetic analogues of $\mathrm{Cu}(\mathrm{II})$ hydroxyl halide minerals have been central to the experimental exploration into the physics of the $S=\frac{1}{2}$ kagomé antiferromagnet in recent years $^{10-12}$. To date, the most widely studied example of this class of materials is the $\mathrm{Zn}$-paratacamite series, $\mathrm{Zn}_{x} \mathrm{Cu}_{4-x}(\mathrm{OH})_{6} \mathrm{Cl}_{2}$, of which the $x=1$ end-member is the QSL candidate herbertsmithite, $\mathrm{ZnCu}_{3}(\mathrm{OH})_{6} \mathrm{Cl}_{2}$. The crystal structure of herbertsmithite contains kagomé layers of $\mathrm{Cu}^{2+} \mathrm{S}=\frac{1}{2}$ moments separated by diamagnetic $\mathrm{Zn}^{2+}$ ions ${ }^{13-15}$, which give rise to a dynamic, disordered magnetic ground state with magnetic moment fluctuations persisting to the lowest measurable temperatures despite strong nearest-neighbour antiferromagnetic exchange $(J=200 \mathrm{~K})^{14,16}$. Precisely characterising the instrinsic physics of the $S=\frac{1}{2}$ kagomé layers of herbertsmithite, however, is extremely challenging due to the presence of $\mathrm{Cu}^{2+} / \mathrm{Zn}^{2+}$ site occupancy disorder within its crystal structure ${ }^{15,17,18}$.

A closely related $\mathrm{Cu}(\mathrm{II})$ hydroxyl halide that has garnered considerable attention in the recent literature is barlowite, $\mathrm{Cu}_{4}(\mathrm{OH})_{6} \mathrm{FBr}^{19}$, a naturally occurring mineral which has been synthetically produced by several groups ${ }^{20-25}$. A recent powder neutron diffraction (PND) study contributed to the ongoing debate around the nuclear and magnetic structures of this material ${ }^{23}$ : like herbertsmithite, barlowite is formed from $\mathrm{Cu}^{2+} \mathrm{S}=\frac{1}{2}$ kagomé layers but with a second interlayer $\mathrm{Cu}^{2+}$ site, which is disordered at roomtemperature in the widely reported $P 6_{3} / \mathrm{mmc}$ model $\mathrm{l}^{20,21,24,25}$. Below $\sim 250 \mathrm{~K}$, one reported class of sample undergoes a subtle structural distortion to an orthorhombic Pnma phase ${ }^{23}$ (see Fig. 1a), which appears to relieve magnetic frustration and allows for the onset of magnetic order at $T_{\mathrm{N}}=15 \mathrm{~K}^{26}$. Intriguingly, computational studies have suggested that barlowite may be tuned towards a QSL phase by substituting the interlayer $\mathrm{Cu}^{2+}$ ions with diamagnetic $\mathrm{Zn}^{2+}$ or

\footnotetext{
${ }^{1}$ Department of Chemistry and Materials Innovation Factory, University of Liverpool, 51 Oxford Street, Liverpool L7 3NY, UK. ${ }^{2}$ Université Paris-Saclay, CNRS, Laboratoire de Physique des Solides, Orsay 91405, France. ${ }^{3}$ James Frank Institute and Department of Physics, University of Chicago, Chicago, IL 60637, USA. ${ }^{4}$ Materials Science Division, Argonne National Laboratory, Argonne, IL 60439, USA. ${ }^{5}$ Laboratory for Muon Spin Spectroscopy, Paul Scherrer Institute, Villigen PSI 5232, Switzerland. ${ }^{6}$ Department of Physics, Durham University, South Road, Durham DH1 3LE, UK. ' ISIS Neutron and Muon Source, Rutherford Appleton Laboratory, Chilton, Didcot, Oxford OX11 0QX, UK. ${ }^{8}$ School of Chemistry,

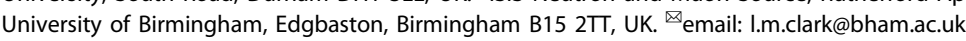



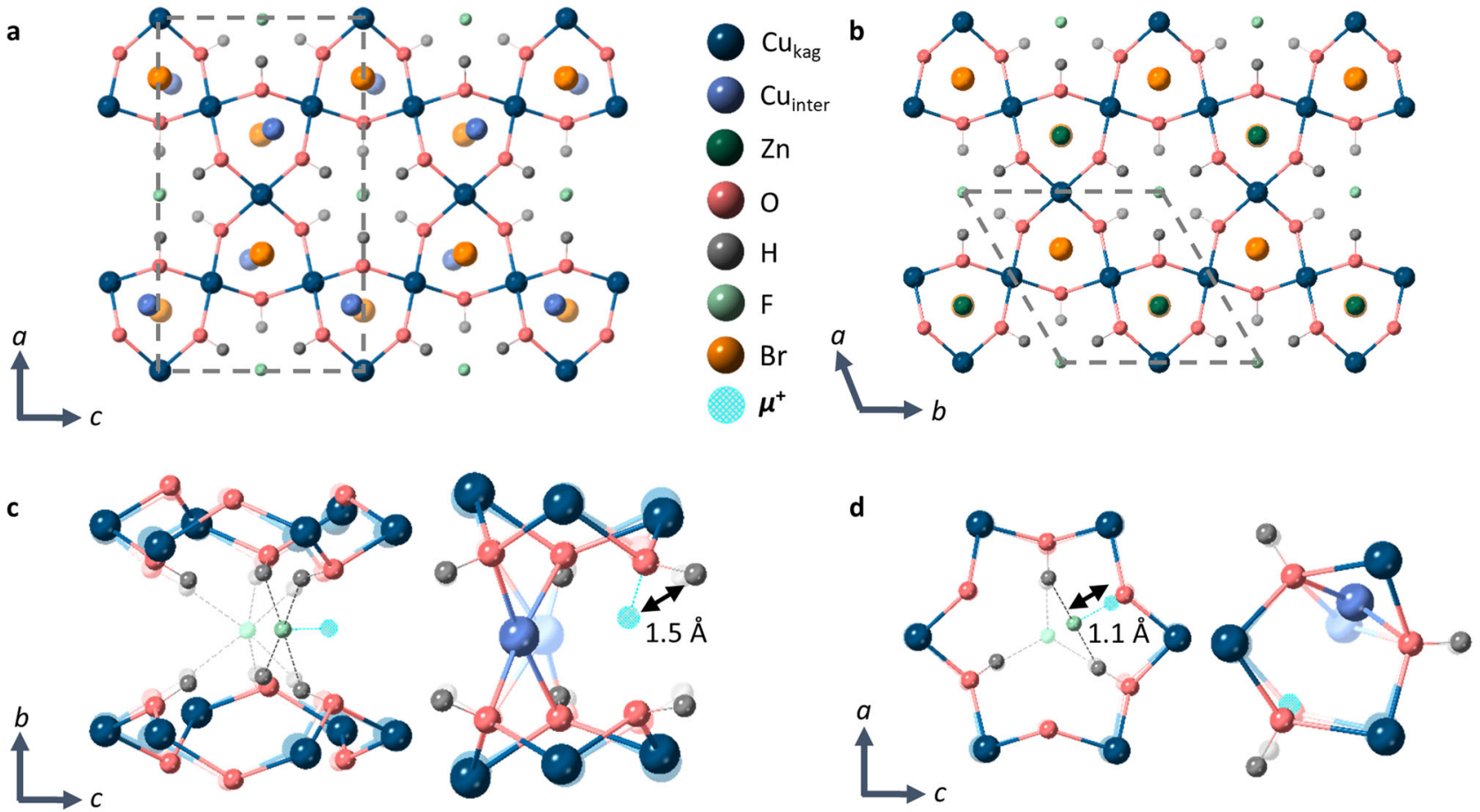

Fig. 1 Low-temperature structures of barlowite and $\mathrm{Zn}$-barlowite. a In barlowite, $\mathrm{Cu}_{4}(\mathrm{OH})_{6} \mathrm{FBr}$, distorted kagome layers---formed from two distinct $\mathrm{Cu}^{2+}$ ions sites $\left(\mathrm{Cu}_{\mathrm{kag}}\right)$ and connected via hydroxide anions---are separated by a third, interlayer $\mathrm{Cu}^{2+}$ ion site $\left(\mathrm{Cu}_{\text {inter }}\right)$ shifted away from the centre of the triangular motifs in the kagome layers above and below. Bromide and fluoride anions also lie between the kagome planes. $\mathbf{b}$ In $\mathrm{Zn}$-barlowite, $\mathrm{ZnCu}_{3}(\mathrm{OH})_{6} \mathrm{FBr}, \mathrm{Zn}^{2+}$ ions separate undistorted kagome layers formed from a single $\mathrm{Cu}^{2+}$ ion site. The two classes of muon stopping sites calculated for barlowite, $\mu-\mathrm{F}$ (left) and $\mu-\mathrm{OH}$ (right), are shown here in the orthorhombic structure viewed along c the $a$-axis, between the kagome layers and $\mathbf{d}$ along the $b$-axis perpendicular to the kagome planes. Faded atoms indicate the unperturbed crystal lattice before muon implantation.

$\mathrm{Mg}^{2+27,28}$ thus reducing the magnetic coupling between the frustrated $S=\frac{1}{2}$ kagomé layers. Furthermore, the calculated energy of formation for cation site occupancy defects is an order of magnitude greater in substituted barlowite than for herbertsmithite ${ }^{27}$, suggesting Mg-barlowite or Zn-barlowite may be highly promising candidates in which to explore the intrinsic properties of a $\mathrm{Cu}^{2+}$-based $\mathrm{S}=\frac{1}{2}$ kagomé antiferromagnet. Indeed, the endmember of the $\mathrm{Zn}$-barlowite series, nominally $\mathrm{ZnCu}_{3}(\mathrm{OH})_{6} \mathrm{FBr}$, has been studied using inelastic neutron scattering and ${ }^{19} \mathrm{~F}$ NMR and seems to display evidence for a gapped $Z_{2}$ QSL $^{29,30}$, a theoretically predicted ground state for the $S=\frac{1}{2}$ Heisenberg model on an antiferromagnetic kagomé net ${ }^{31}$.

Despite this promise, the evolution of the magnetic ground state and characteristic magnetic moment correlation dynamics across the $\mathrm{Zn}$-barlowite series are yet to be explored in any great detail using local probe techniques, such as muon spin relaxation and rotation $(\mu \mathrm{SR})$. In these techniques, spin-polarised, positively charged muons are implanted into a sample of interest, in which they thermalise in regions of high electron density. From there, the spin polarisation of implanted muons will evolve over time, depending on the presence of local magnetic fields at the muon stopping site, which may originate from both nuclear and electronic moments within the sample. Following the timedependence of the muon spin polarisation-via the asymmetry of the emitted muon-decay positrons-thus provides a unique insight into the local magnetic properties of condensed matter systems ${ }^{32}$. As the muon spin is sensitive to very small magnetic fields $\left(\sim 10^{-5} \mathrm{~T}\right)$ and fluctuating field dynamics on a microsecond timescale, $\mu \mathrm{SR}$ has proved to be invaluable in revealing dynamical magnetic correlations in herbertsmithite and many other frustrated quantum magnets ${ }^{10,16,33-37}$. However, limitations in the interpretation of $\mu \mathrm{SR}$ data often stem from the lack of knowledge of the possible muon stopping sites within complex crystal structures and the extent to which the implanted muons distort the surrounding lattice ${ }^{38}$. Crucially, the development of densityfunctional theory (DFT) to calculate muon stopping sites in crystalline solids is increasingly enabling the determination of these factors to advance the quantitative analysis of experimental $\mu \mathrm{SR}$ data ${ }^{39}$. Here, we present such a study-combining comprehensive $\mu S R$ measurements on the $Z n$-barlowite series with supporting DFT muon-site calculations-to reveal the onset of the possible QSL phase within this family of quantum materials. In this case, we take advantage of the formation of both $\mu-\mathrm{F}$ and $\mu-\mathrm{OH}$ complexes upon muon implantation in $\mathrm{Zn}$-barlowite which - in combination with our DFT calculations - gives a strong constraint for the accurate determination of muon stopping sites and the local magnetic properties of this series.

\section{RESULTS AND DISCUSSION}

Combining zero-field $\mu$ SR measurement and DFT to identify muon stopping sites in barlowite

We first consider the parent compound barlowite, $\mathrm{Cu}_{4}(\mathrm{OH})_{6} \mathrm{FBr}$, which-in accordance with previous studies ${ }^{20-22,24}$-orders at $T_{\mathrm{N}}=15 \mathrm{~K}$ as indicated by the sharp upturn in the magnetic susceptibility shown in Fig. 2a. Curie-Weiss fitting the hightemperature inverse susceptibility (shown in Supplementary Fig. 1) yields an antiferromagnetic Weiss temperature, $\theta=-111.4(3) \mathrm{K}$, which is manifested in the antiferromagnetic alignment of $\mathrm{Cu}^{2+}$ moments within the kagomé planes of barlowite in its magnetic structure previously determined by neutron diffraction ${ }^{23}$. This magnetic structure includes a ferromagnetic component due to the canting of the magnetic moments at the interlayer $\mathrm{Cu}^{2+}$ site. Figure $3 a$ shows the muon decay asymmetry measured in 
zero-field (ZF) on the MuSR spectrometer for such a sample. Above $T_{\mathrm{N}}$, the long-time oscillations observed in the data are characteristic of the local nuclear magnetic fields at the muon stopping sites within the sample. In particular, such oscillations are indicative of dipolar coupling between the muon and nuclear spins that leads to the formation of entangled state complexes. DFT muon-site
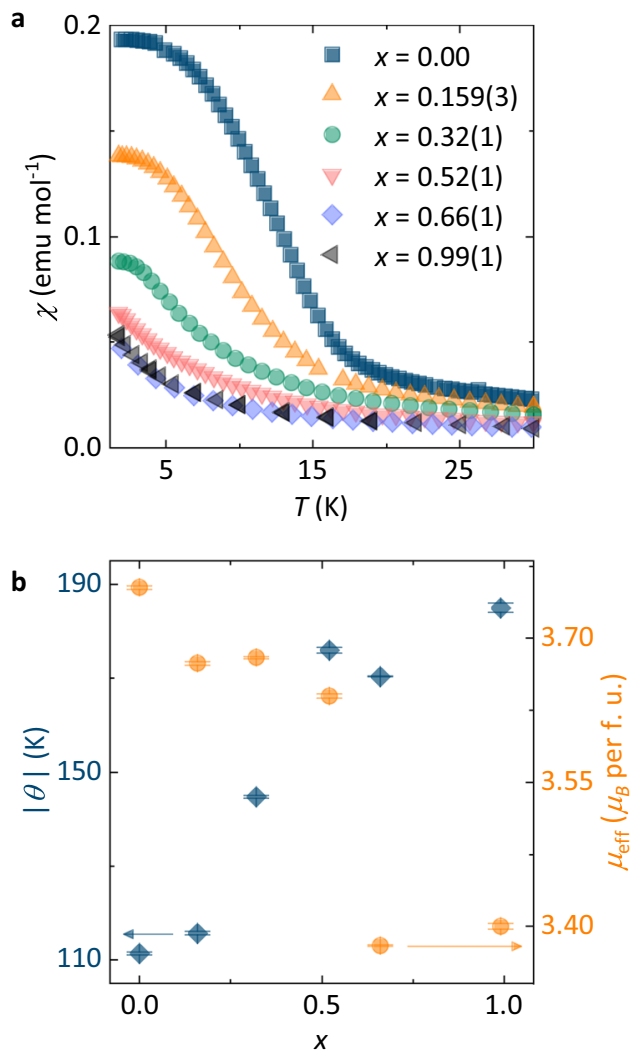

Fig. 2 Magnetic susceptibility of $\mathrm{Zn}_{x} \mathrm{Cu}_{4-x}(\mathrm{OH})_{6} \mathrm{FBr}$. a The temperature-dependent magnetic susceptibility measured in a $1 \mathrm{~T}$ field with zero-field cooling. b The absolute values of the antiferromagnetic Weiss constants, $\theta$ (left axis), and the effective magnetic moments, $\mu_{\text {eff }}$ (right axis), obtained from Curie-Weiss fitting the inverse magnetic susceptibility for all compositions, $x$, as shown in Supplementary Fig. 1. Error bars in $\mathbf{b}$ represent the standard error of the fit parameters, $\theta$ and $\mu_{\text {eff. }}$ calculations reveal two distinct classes of muon stopping site complexes in barlowite, which we show are consistent with the experimental ZF asymmetry signal shown in Fig. 3a. The first class of muon stopping site localises $\sim 1.0 \AA$ away from the oxygen atoms in the hydroxide groups that connect the $\mathrm{Cu}^{2+}$ ions within the kagomé layers of barlowite. This forms a triangular $\mu-\mathrm{OH}$ complex, with the muon-proton distance found to be $1.54 \AA$ in the lowest-energy sites, as shown in Fig. 1c. In the second class of muon stopping site, muons are found to localise near the fluoride anions in between the kagomé layers of barlowite, with a $\mu-\mathrm{F}$ separation of $1.1 \AA$, as depicted in Fig. $1 \mathrm{~d}$. Such $\mu-\mathrm{F}$ sites lie at substantially higher energies above the lowest energy $\mu-\mathrm{OH}$ sites in our calculations $(\approx 1 \mathrm{eV})$. Although this suggests-on purely energetic grounds - that the formation of $\mu-\mathrm{F}$ complexes in barlowite is unlikely compared to $\mu-\mathrm{OH}$, it is possible that during the stopping process, muons can be captured in these local potential minima.

Based on the results of these DFT calculations, we have devised a model that describes the ZF asymmetry of barlowite in the paramagnetic regime, incorporating the two distinct classes of muon stopping sites,

$$
\begin{aligned}
A(t)= & f_{F} P_{F}(t) \exp \left(-\frac{\gamma_{\mu}^{2} \Delta_{F}^{2} t^{2}}{2}\right)+f_{O H} P_{O H}(t) \exp \left(-\frac{\gamma_{\mu}^{2} \Delta_{O H}^{2} t^{2}}{2}\right) \\
& +A_{b g} \exp \left(-\lambda_{b g} t\right) .
\end{aligned}
$$

$A_{\mathrm{bg}}$ accounts for the background contribution of muons that stop outside the sample, which is weakly relaxing, and the expressions $P_{\mathrm{F}}(t)$ and $P_{\mathrm{OH}}(t)$ describe the dipolar interactions between the nuclear magnetic moments of fluoride and hydroxide groups and the muon spin at each class of stopping site within the sample, given by ${ }^{40}$,

$P_{F(O H)}(t)=\frac{1}{6}+\frac{1}{3} \cos \left(\frac{\omega_{F(O H)} t}{2}\right)+\frac{1}{6} \cos \left(\omega_{F(O H)} t\right)+\frac{1}{3} \cos \left(\frac{3 \omega_{F(O H)} t}{2}\right)$.

The oscillation frequencies $\omega_{\mathrm{F}}$ and $\omega_{\mathrm{OH}}$ are related to the respective $\mu-\mathrm{F}$ and $\mu-\mathrm{H}$ distances, $d_{\mathrm{F}(\mathrm{H})}$, through,

$\omega_{F(O H)}=\frac{\mu_{0} \hbar \gamma_{\mu} \gamma_{F(H)}}{4 \pi d_{F(H)}^{3}}$

where $\mu_{0}$ is the permeability of free space and $\gamma_{\mu}=2 \pi \times$ $135.5 \mathrm{MHz} \mathrm{T}^{-1}, \gamma_{\mathrm{F}}=2 \pi \times 40.1 \mathrm{MHz} \mathrm{T}^{-1}$ and $\gamma_{\mathrm{H}}=2 \pi \times 42.6 \mathrm{MHz} \mathrm{T}^{-1}$ are the gyromagnetic ratios of the muon spin and the fluorine and hydrogen nuclear spins, respectively. A fit of this model to ZF data
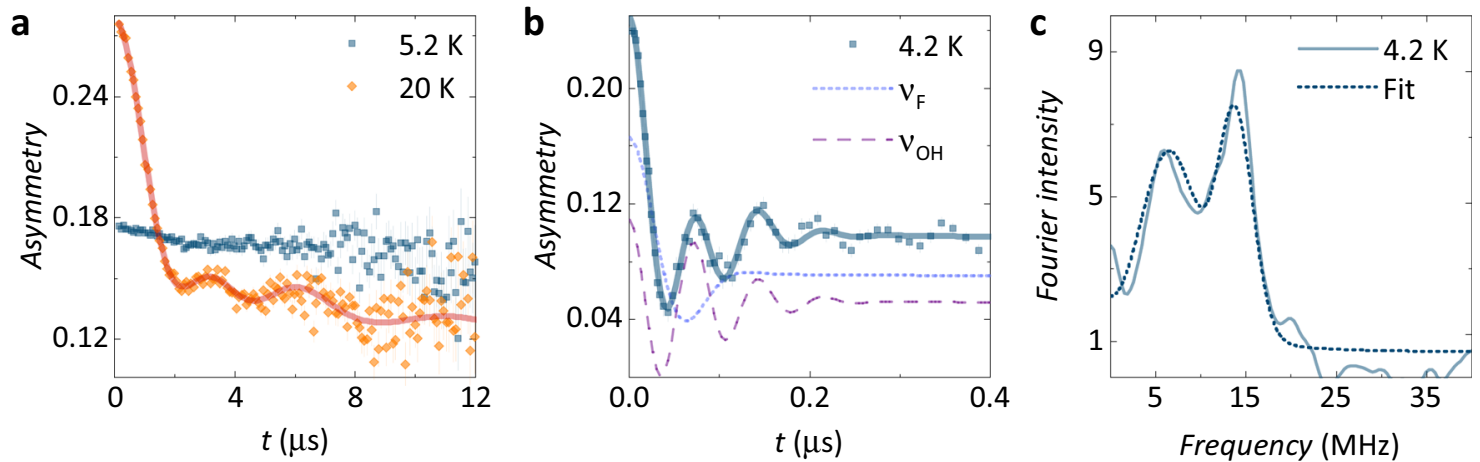

Fig. 3 Time-dependent zero-field muon asymmetry for $\mathrm{Cu}_{4}(\mathbf{O H})_{6} \mathbf{F B r}$. a Data measured at 5.2 and $20 \mathrm{~K}$ on the MuSR spectrometer, with the solid line showing the fit of Equation 1 to the high-temperature data. $\mathbf{b}$ Data collected at $4.2 \mathrm{~K}$ on the GPS instrument, which can be modelled using Eq. 4, as shown by the solid line. The dashed lines indicate the two distinct oscillation frequencies contributing to the model. c The Fourier transform of the time-dependent signal shown in part $\mathbf{b}$ clearly reveals these two frequencies at $v_{\mathrm{F}}=6.3(2) \mathrm{MHz}$ and $v_{\mathrm{OH}}=13.7(2)$ $\mathrm{MHz}$, corresponding to local dipolar fields, $B_{F}^{\text {loc }}=46(2) \mathrm{mT}$ and $B_{O H}^{\text {loc }}=101(2) \mathrm{mT}$. Error bars in (a) and (b) represent one standard deviation. 
Table 1. Muon stopping sites in $\mathrm{Cu}_{4}(\mathrm{OH})_{6} \mathrm{FBr}$.

\begin{tabular}{|c|c|c|c|c|c|}
\hline \multicolumn{2}{|c|}{$\overline{\mu^{+} \text {Stopping Site }}$} & \multicolumn{3}{|c|}{$\omega\left(\mathrm{Mrad} \mathrm{s}^{-1}\right)$} & $\Delta(\mathrm{mT})$ \\
\hline \multicolumn{2}{|l|}{$\mathrm{F}^{-}$} & \multicolumn{3}{|c|}{$1.23(2)$} & $0.43(4)$ \\
\hline \multicolumn{3}{|l|}{$\mathrm{OH}^{-}$} & \multicolumn{2}{|c|}{$0.72(2)$} & $0.18(4)$ \\
\hline \multicolumn{2}{|l|}{$f(\%)$} & \multicolumn{2}{|l|}{$d(\AA \AA)$} & \multicolumn{2}{|c|}{$B^{\text {loc }}(\mathrm{mT})$} \\
\hline MuSR & GPS & MuSR & DFT & GPS & Calculated \\
\hline $69(4)$ & $64(2)$ & $1.22(1)$ & 1.11 & $46(2)$ & 55 \\
\hline 31 (4) & $34(2)$ & $1.50(3)$ & 1.54 & $101(2)$ & 120 \\
\hline
\end{tabular}

Top: Parameters obtained from fitting Eq. 1 to zero-field, $20 \mathrm{~K}$ MuSR data (Fig. 3a). Bottom: The fraction of muons, $f$, stopping at each class of site, as determined from MuSR (Eq. 1, Fig. 3a) and GPS data (Eq. 4, Fig. 3b) as well as the comparison between experiment and calculation for the $\mu-\mathrm{F}(\mathrm{H})$ bond lengths, $d$, and their local dipolar fields, $\boldsymbol{B}_{\text {loc }}$, below $T_{\mathrm{N}}$.

collected for barlowite is shown in Fig. 3a with the fitting parameters given in Table 1. The experimentally determined $\mu-\mathrm{F}$ and $\mu-\mathrm{H}$ distances are in good agreement with those calculated from DFT. The Gaussian damping term in Equation 1 phenomenologically describes a possible distribution of nuclear fields surrounding the muon spin, with $\Delta_{\mathrm{F}}$ and $\Delta_{\mathrm{OH}}$ describing the field distributions at each class of stopping site. Indeed, DFT calculations also suggest that there are many possible stopping sites within the $\mu-\mathrm{OH}$ class, separated in total energy by $<0.1 \mathrm{eV}$. This reflects the possibility that muons localise near the oxygen atoms in a number of similar configurations. There is some variation in the $\mu-\mathrm{H}$ distances calculated for these similar stopping sites, with $1.63 \AA$ found in a few of the higher-energy sites in this class. In all calculated cases of $\mu-\mathrm{OH}$ complex, however, the muon causes a distortion to the local crystal structure in the vicinity of its stopping site. In the lowest-energy sites of this class, the muon pushes the closest $\mathrm{Cu}^{2+}$ ion away from the muon site by $\sim 0.45 \AA$, as demonstrated in Fig. 1c and d. In contrast, little distortion of the local environment occurs close to the $\mu-\mathrm{F}$ sites.

Resolving magnetic order in barlowite through high-resolution $\mu \mathrm{SR}$

Below $T_{N}$, the internal electronic magnetic fields arising from the ordered $\mathrm{Cu}^{2+}$ moments in barlowite rapidly depolarise the implanted muon spins. As a consequence, much of the initial asymmetry in our MuSR datasets is lost, as can be seen in Fig. 3a, owing to the limited time-resolution of the pulsed muon beam at the ISIS Neutron and Muon Source. We do, however, observe a 'one-third tail' in the baseline asymmetry, a feature attributed to the presence of static magnetic moments in a polycrystalline sample, where on average, one-third of the implanted muon spins will be initially parallel to the local magnetic field, and whose polarisation will thus remain unaffected in the absence of dynamics ${ }^{41}$. To overcome this limitation, we turn to data collected for barlowite on the GPS instrument at PSI, shown in Fig. 3b and Supplementary Fig. 4, for which the continuous nature of the muon source provides sufficient time-resolution to capture the spontaneous oscillations apparent below $0.25 \mu \mathrm{s}$ in the ZF asymmetry signal. These oscillations are indicative of the muon spin precession around the static internal magnetic fields of electronic origin at a frequency given by $v_{i}=\left(\gamma_{\mu} / 2 \pi\right) B_{i}^{\text {loc }}$, where $B_{i}^{\text {loc }}$ is the local magnetic field at each muon stopping site, $i$, arising from the neighbouring ordered $\mathrm{Cu}^{2+}$ moments. Consistent with the DFT calculations and the analysis of the ZF asymmetry data collected above $T_{\mathrm{N}}$, the spontaneous oscillations in the ZF asymmetry data collected below $T_{\mathrm{N}}$ in Fig. $3 \mathrm{~b}$ can also be modelled in terms of two distinct classes of muon stopping site with the following expression,

$$
A(t)=\sum_{i=1}^{2} a_{i}\left[\frac{2}{3} \cos \left(2 \pi v_{i}+\phi\right) \exp \left(-\sigma_{i}^{2} t^{2}\right)+\frac{1}{3} \exp (-\lambda t)\right]+A_{b g} \text {. }
$$

The two dominant oscillating frequencies, $v_{1}=6.3(2) \mathrm{MHz}$ and $v_{2}=13.7(2) \mathrm{MHz}$, extracted from this fit can also be clearly resolved in the Fourier transform of the GPS data collected at $4.2 \mathrm{~K}$, as shown in Fig. 3c. Comparing the fitted amplitudes of each component, $a_{1}=64(2) \%$ and $a_{2}=34(2) \%$, with the stopping site fractions, $f_{\mathrm{F}}$ and $f_{\mathrm{OH}}$, obtained from fitting the ZF data above $T_{\mathrm{N}}$ in Fig. $3 a$ (see Table 1), we assign the former to the $\mu-\mathrm{F}$ and the latter to the $\mu-\mathrm{OH}$ classes of muon stopping site. From this assignment, the data imply that the local magnetic fields at each class of muon stopping site arising from neighbouring ordered $\mathrm{Cu}^{2+}$ moments are thus $B_{F}^{\text {loc }} \approx 46 \mathrm{mT}$ and $B_{O H}^{\text {loc }} \approx 101 \mathrm{mT}$, respectively. It should be noted that the weak relaxation of the one-third tail in the asymmetry data should not be mistaken for residual spin dynamics in barlowite below $T_{\mathrm{N}}$. In the case of Fig. $3 \mathrm{~b}$, it is due to the small fraction of muons that stop in the aluminium foil used to contain the sample, whose spins thus experience the nuclear fields of ${ }^{27} \mathrm{Al}$.

As a final consistency check for our combination of DFT methods and muon experiment, we estimate the local electronic magnetic moment dipolar fields at each of the calculated muon stopping site classes based on the known magnetic structure for barlowite below $T_{\mathrm{N}}{ }^{23}$. For the lowest-energy $\mu-\mathrm{OH}$ stopping sites, and assuming an undistorted crystal structure, these calculations suggest typical local fields around $120 \mathrm{mT}$, along with a few around $75 \mathrm{mT}$. If the muon-induced distortion is taken into account we obtain still smaller fields on the order of $50 \mathrm{mT}$, owing to the movement of the closest $\mathrm{Cu}^{2+}$ moment. The $B_{O H}^{\text {loc }}$ fields obtained are, therefore, highly sensitive to this distortion, but it is probable that this effect is over estimated in our computations owing to the relatively small size of the supercell used. The average $B_{F}^{\text {loc }}$ for the $\mu-F$ class of stopping sites is smaller, at typically $55 \mathrm{mT}$, and these values are insensitive to the inclusion of the distortions. Overall, the agreement of these calculations with the fields determined from our PSI data (summarised in Table 1) reinforces the likely formation of $\mu-\mathrm{F}$ stopping sites in barlowite despite their overall higher energy compared with $\mu-\mathrm{OH}$.

Following the evolution towards quantum disorder in $\mathrm{Zn}$ barlowite through transverse-field (TF) $\mu \mathrm{SR}$

We next consider the effect of substituting $\mathrm{Cu}^{2+} \mathrm{S}=\frac{1}{2}$ ions in barlowite for diamagnetic $\mathrm{Zn}^{2+}$ in the series, $\mathrm{Zn}_{x} \mathrm{Cu}_{4-x}(\mathrm{OH})_{6} \mathrm{FBr}$. Our analysis of PND data collected for $\mathrm{Zn}$-substituted samples, shown in Supplementary Fig. 2 and Supplementary Table 3, yields two important observations. First, for highly substituted samples $(x>0.5)$ the crystal structure retains hexagonal $P 6_{3} / m m c$ symmetry down to $1.5 \mathrm{~K}$-the base temperature of our diffraction experiments-in which the kagomé layers remain undistorted. Second, refinement of the cation site occupancies within this hexagonal structure indicate that the substituted $\mathrm{Zn}^{2+}$ ions predominately occupy the trigonal prismatic interplane site, with only $0.6(2) \%$ of the intralayer sites occupied by $\mathrm{Zn}^{2+}$, an order of magnitude less than reported for herberstmithite ${ }^{17}$. This is in keeping with theory ${ }^{28}$ and suggests that $\mathrm{Zn}$-substitution effectively reduces the magnetic coupling between the kagomé layers of $\mathrm{Cu}^{2+}$ moments in barlowite. Correspondingly, we observe a progressive suppression of the magnetic ordering transition in the temperaturedependent magnetic susceptibility for $\mathrm{Zn}_{x} \mathrm{Cu}_{4-x}(\mathrm{OH})_{6} \mathrm{FBr}$ with increasing $x$, as shown in Fig. 2a. Figure $2 \mathrm{~b}$ shows the fitting parameters obtained from the Curie-Weiss analysis of these data, revealing a reduction in the effective magnetic moment, $\mu_{\text {eff, }}$ per formula unit across the series as $\mathrm{Cu}^{2+}$ is replaced with $\mathrm{Zn}^{2+}$, and 
an increase in the absolute value of the Weiss constant, $\theta$, since the net magnetic exchange becomes increasingly negative as the positive ferromagnetic contribution from the interlayer coupling is reduced across the series.

In order to follow the evolution of the magnetic ground state in Zn-barlowite, we performed a set of TF $\mu$ SR measurements across the series, in which a magnetic field of $2 \mathrm{mT}$ is applied perpendicular to the initial muon spin polarisation. In this TF geometry for a sample in the paramagnetic regime, implanted muon spins precess around the applied field with a frequency, $v_{\mathrm{TF}}$. Below $T_{N}$, however, the local internal magnetic fields within the magnetically ordered state can dominate the muon spin relaxation, resulting in a loss of asymmetry and a dephasing of the oscillating signal measured in the TF experiment. Both of these effects can be seen in the TF data collected for an $x=0.00$ sample above and below $T_{\mathrm{N}}$ shown in Fig. $4 \mathrm{a}$, and can be modelled by,

$$
A(t)=\left[A_{p} \exp \left(-\lambda_{T F} t\right)+A_{b g} \exp \left(-\sigma_{b g}^{2} t^{2}\right)\right] \times \cos \left(2 \pi v_{T F} t+\phi\right)+B,
$$

where $A_{\mathrm{p}}$ provides a measure of the paramagnetic (dynamic) volume fraction of the sample, $A_{\mathrm{bg}}$ gives the background contribution of muons that stop in the silver sample holder and $B$ accounts for the fraction of muons with their spin polarisation aligned with the local magnetic fields in the ordered state of our polycrystalline samples. Therefore, in the $x=0.00$ sample below $T_{N}, B$ tends to one-third of the asymmetry we observe in the paramagnetic state, in a similar manner to the one-third tail observed in our ZF data discussed above. By fitting this model to TF data collected over a range of temperatures, we may plot the temperature dependence of $A_{\mathrm{p}}$ or, in this case, the normalised
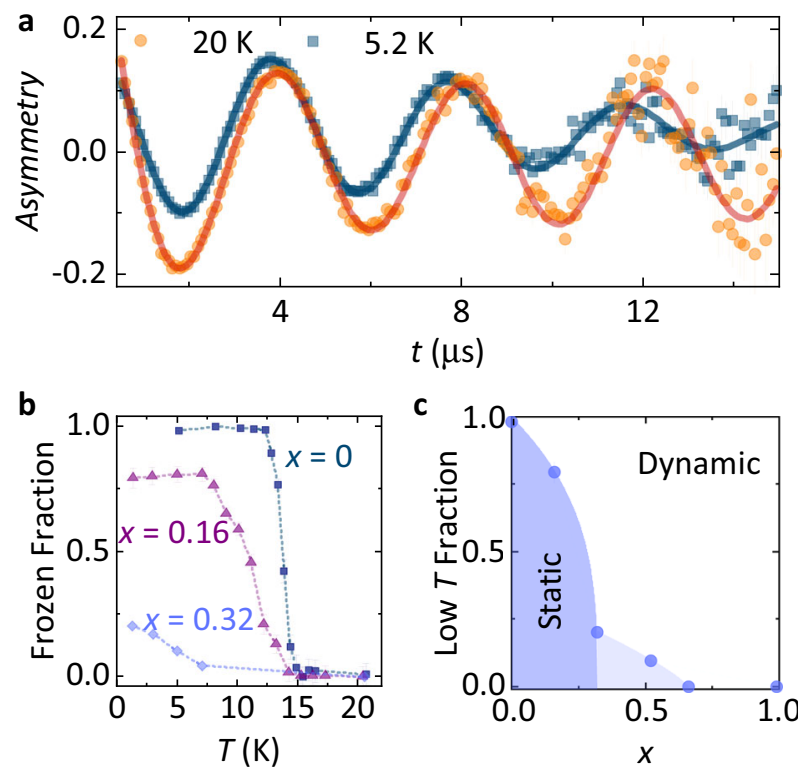

Fig. 4 Time-dependent transverse-field muon asymmetry for $\mathrm{Cu}_{4}(\mathrm{OH})_{6} \mathrm{FBr}$. a Asymmetry measured in a $2 \mathrm{mT}$ transverse-field at 5.2 and $20 \mathrm{~K}$. The solid lines are fits of Eq. 5 to the data. Error bars represent one standard deviation. b Frozen fraction of static $\mathrm{Cu}^{2+}$ moments as a function of temperature determined from transversefield data collected for a range of sample compositions, $\mathrm{Zn}_{x} \mathrm{Cu}_{4-x}$ $(\mathrm{OH})_{6} \mathrm{FBr}$. The dashed lines are guides to the eye. c The lowtemperature frozen fraction as a function of $x$, where the dark shaded region highlights the critical-like fall-off of static $\mathrm{Cu}^{2+}$ moments upon the $\mathrm{Zn}^{2+}$ substitution of interlayer sites. The lightly shaded region indicates a crossover regime between static and dynamic ground states. Error bars in (b) and (c) represent the propagated standard error of the fit parameter, $A_{\mathrm{p}}$. inverse of this value (i.e. $1-A_{\mathrm{p}}(T) / A_{\mathrm{p}}(20 \mathrm{~K})$ ), which represents the evolution of the frozen volume fraction of static magnetic moments within a sample. For example, for the $x=0.00$ sample, a sudden drop in $A_{\mathrm{p}}$ at $T_{\mathrm{N}}=15 \mathrm{~K}$ corresponds to a dramatic increase in the static volume fraction, shown in Fig. 4b, a clear signal of the magnetic ordering transition, in agreement with our magnetic susceptibility (Fig. 2a) and PND measurements ${ }^{23}$.

The same analysis can be applied to samples in the $\mathrm{Zn}$ barlowite series upon increasing $\mathrm{Zn}^{2+}$ content, and Fig. $4 \mathrm{~b}$ shows the temperature dependence of the frozen volume fraction for $\mathrm{Zn}_{x} \mathrm{Cu}_{4-x}(\mathrm{OH})_{6} \mathrm{FBr}$ with $x=0.159(3)$ and $0.32(1)$. For $x=0.159(3)$ we observe an extended magnetic ordering regime, while for the $x=0.32$ (1) sample, the static moment fraction at base temperature is vastly reduced compared with the parent $x=0.00$ compound. Moreover, there is also evidence to support the coexistence of static and dynamic magnetic correlations in the $x=0.159(3)$ and $0.32(1)$ members of the $\mathrm{Zn}$-barlowite series, as in both ZF and TF measurements of these samples we do not recover the one-third asymmetry expected for a fully ordered magnetic ground state in a polycrystalline sample, as shown in Supplementary Fig. 3. As the $\mathrm{Zn}^{2+}$ content of $\mathrm{Zn}_{x} \mathrm{Cu}_{4-x}(\mathrm{OH})_{6} \mathrm{FBr}$ is increased further to $x=0.52(1)$, dynamic magnetic correlations dominate the ZF muon asymmetry at low-temperature-likely indicating a static to dynamic crossover region in the magnetic phase diagram-and by $x=0.66(1)$, the magnetic moments of the $\mathrm{Cu}^{2+} \mathrm{S}=\frac{1}{2}$ ions remain dynamically fluctuating down to the lowest temperatures of our $\mu \mathrm{SR}$ experiment. This is most clearly evidenced by the low-temperature ZF data collected for the $x=0.66(1)$ sample shown in Fig. $5 a$, which can be modelled using
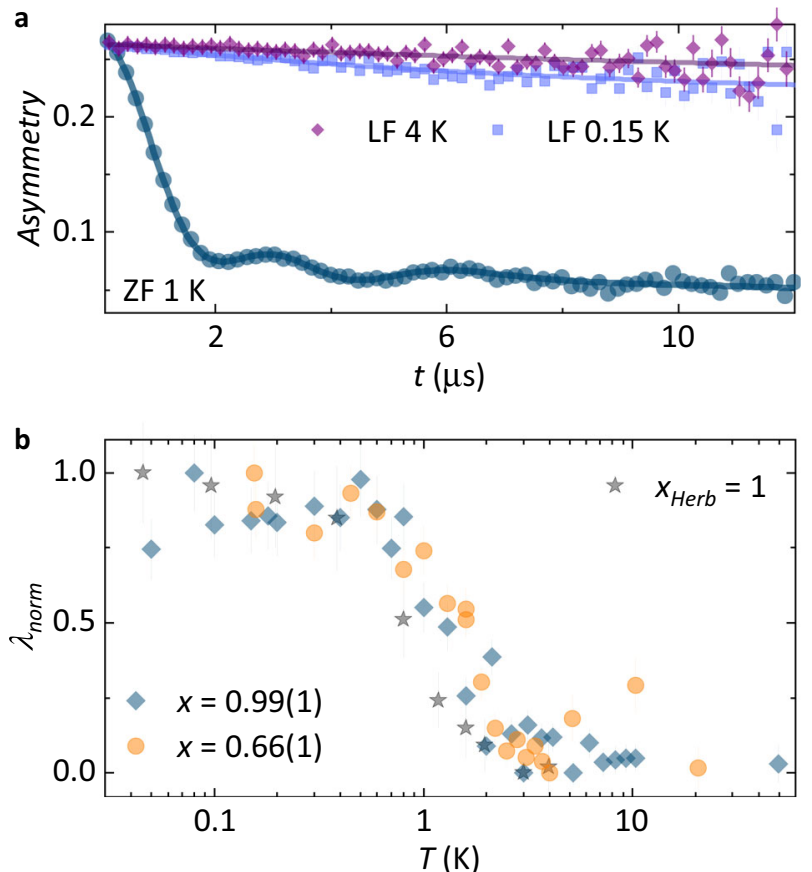

Fig. 5 Persistent dynamics in $\mathbf{Z n}$-barlowite. a Time-dependent muon asymmetry measured for $\mathrm{Zn}_{0.66(1)} \mathrm{Cu}_{3.34}(\mathrm{OH})_{6} \mathrm{FBr}$ in zero-field at $1 \mathrm{~K}$, where the solid line shows a fit using Eq. 1, and in an applied longitudinal field of $10 \mathrm{mT}$ at 0.15 and $4 \mathrm{~K}$, where solid lines show fits of Eq. 6 to the data. Error bars represent one standard deviation. b Temperature dependence of the normalised muon spin relaxation rate, $\lambda_{\text {norm }}$ obtained from longitudinal-field data collected for $x=$ $0.66(1)$ and $x=0.99$ (1) samples. As a comparison, data points for $x=$ 1.0 herbertsmithite, $\mathrm{ZnCu}_{3}(\mathrm{OH})_{6} \mathrm{Cl}_{2}$, obtained from ref. ${ }^{16}$, are also shown. Error bars in (b) represent the standard error of the fit parameter, $\lambda$. 
the same expression developed for the $x=0.00$ compound in the paramagnetic regime (Eq. 1 and Supplementary Table 4). An important result from our DFT calculations in this regard is that the inclusion of $\mathrm{Zn}^{2+}$ ions and the hexagonal space group symmetry of the $\mathrm{Zn}$-substituted samples does not yield any notably different classes of low-energy muon stopping sites compared with the parent orthorhombic structure. Together, our observations from ZF and TF $\mu$ SR measurements from across the Zn-barlowite series allow us to map out the low-temperature magnetic phase diagram shown in Fig. 4c, which reveals a critical reduction in the static volume fraction from below $x=0.5$ as the system enters a possible QSL state. This is strikingly similar to the magnetic phase diagram known for the $\mathrm{Zn}$-paratacamite series ${ }^{16}$, in which the static volume fraction drops to zero near $x=0.6$, and is reproduced well by a recent study of the $\mathrm{Zn}$-barlowite phase diagram by Wei et al. ${ }^{42}$.

Probing spin fluctuations in Zn-barlowite through longitudinalfield $\mu \mathrm{SR}$

Finally, we turn to explore the dynamical correlations within the quantum disordered ground state of $\mathrm{Zn}_{x} \mathrm{Cu}_{4-x}(\mathrm{OH})_{6} \mathrm{FBr}$ for $x \geq$ 0.66 . In order to isolate and probe the dynamics of the electronic magnetic moment fluctuations in these highly substituted samples, we applied a longitudinal field (LF) of $10 \mathrm{mT}$ in our $\mu \mathrm{SR}$ experiments. In doing so, the implanted muon spins are decoupled from the nuclear magnetic moments that dominate the ZF signal, meaning any remaining depolarisation of the muon spin arises from its interaction with the local electronic magnetic fields (see Supplementary Fig. 5). Figure 5a shows representative LF data for the $x=0.66(1)$ sample, which can be modelled at all temperatures using a simple exponential relaxation function,

$A(t)=A_{r} \exp (-\lambda t)+A_{b g}$

where $\lambda$ is the muon spin relaxation rate, $A_{r}$ is the relaxing amplitude and $A_{\mathrm{bg}}$ is the background contribution from muons stopping outside the sample. Such an exponential relaxation of the LF signal of a sample is characteristic of electronic magnetic moment fluctuations within a motional narrowing regime, with the corresponding relaxation rate described by the Redfield equation ${ }^{43}$ :

$\lambda=\frac{2 \gamma_{\mu}^{2} \Delta^{2} \tau}{1+\gamma_{\mu}^{2} B_{L F}^{2} \tau^{2}}$

where $\gamma_{\mu}$ is the muon spin gyromagnetic ratio, $B_{\mathrm{LF}}$ is the applied longitudinal-field and $\Delta$ and $\tau$ are the internal electronic magnetic field distribution and its fluctuation time-characteristic to a particular sample-respectively. A common feature that emerges from the analysis of LF $\mu$ SR data of many QSL candidates of diverse chemical and structural varieties is the presence of a plateau in the muon spin relaxation rate, $\lambda$, at lowtemperatures ${ }^{16,37,44-47}$.

Qualitatively, this can be taken to indicate the presence of a QSL ground state characterised by dynamic electronic magnetic moment fluctuations with a temperature-independent fluctuation time, which is unlike any system with a static magnetic ground state, for example, a spin glass, which is associated with a maximum in $\lambda$ near its spin freezing temperature ${ }^{48}$. Quantitatively, however, it is challenging to meaningfully compare muon spin relaxation rates observed in the plateau states across different classes of QSL candidate materials because, as Eq. 7 highlights, there are several key parameters that will determine the overall magnitude of $\lambda$ for any given system, as well as experimental factors, such as the applied LF and signal background. Even across a single family of materials-here $\mathrm{Zn}_{x} \mathrm{Cu}_{4-x}(\mathrm{OH})_{6} \mathrm{FBr}$-substituting $\mathrm{Cu}^{2+}$ for diamagnetic $\mathrm{Zn}^{2+}$ will affect the size and distribution of the internal electronic magnetic field, which can have pronounced consequences for the magnitude of $\lambda$. For instance, in this particular case, one might expect that as $x$ increases across the $\mathrm{Zn}_{x} \mathrm{Cu}_{4-x}(\mathrm{OH})_{6} \mathrm{FBr}$ series at a given temperature, $\Delta$ will decrease as magnetic $\mathrm{Cu}^{2+}$ is replaced by diamagnetic $\mathrm{Zn}^{2+}$, but that $\tau$ will increase as the system approaches a dynamic spin liquid state. Together, this implies that the magnitude of $\lambda$ will decrease with increasing concentration of Zn-substitution, which has been observed in the Zn-paratacamite family of herbertsmithite ${ }^{16}$, but does not appear to be the case for samples of $Z n$-barlowite with $x$ $>0.5$ (see Supplementary Fig. 6). However, another compelling viewpoint to consider in this regard is that at low-temperatures, muon spins implanted within highly substituted samples of $\mathrm{Zn}$ barlowite may be predominantly influenced by their dipolar interaction with defect spins of $\mathrm{Cu}^{2+}$ moments remaining at the interlayer sites ${ }^{49}$. If this is the case, then one would expect a decrease in the dominant dipolar fields coupling to the muon spin as the concentration of defect $\mathrm{Cu}^{2+}$ moments decreases with increasing Zn-subsitution. Such an argument could thus account for the observed variation in the magnitude of $\lambda$ in $Z n$-barlowite for $x>0.5$, and further supports the notion that cation site occupancy defects are reduced in substituted barlowite compared with herbertsmithite owing to the differences in their crystal strucures ${ }^{27}$.

However, for members of the Zn-barlowite series with $x>0.5-$ for which we have demonstrated that magnetic order is suppressed-one would not expect the internal field distribution, $\Delta$, within a particular sample to vary significantly with temperature. Therefore, by normalising the muon spin relaxation rates obtained for highly substituted members of the Zn-barlowite series, shown in Supplementary Fig. 6, we can directly compare their dynamical magnetic moment correlations across the series, which will be primarily governed by their temperature-dependent field fluctuation time, $\tau$. Figure $5 b$ shows the comparison of the normalised relaxation rate, $\lambda_{\text {norm }}$, for $\mathrm{Zn}_{x} \mathrm{Cu}_{4-x}(\mathrm{OH})_{6} \mathrm{FBr}$ with $x=$ $0.66(1)$ and $0.99(1)$. Both samples have a prominent plateau in the muon spin relaxation that persists to the lowest measured temperatures-in the case of the $x=0.99$ (1) sample, to at least $50 \mathrm{mK}$-indicating an absence of any ordering or freezing of $\mathrm{Cu}^{2+}$ magnetic moments despite the strong exchange interactions between them $(|\theta| \approx 190 \mathrm{~K}$ for $x=0.99(1)$, (see Fig. 2b). Our observations are thus in support of the recent proposals of a QSL ground state for fully subsituted $\mathrm{ZnCu}_{3}(\mathrm{OH})_{6} \mathrm{FBr}$ from both experiment and theory ${ }^{30,50}$. Another important result from our present study, however, is the striking similarity of the temperature dependence of $\lambda_{\text {norm }}$ for $x=0.66(1)$ and $0.99(1)$ samples, as seen in Fig. 5b. This suggests that the nature of the dynamical field fluctuations in highly substituted $\mathrm{Zn}$-barlowite-and its potential QSL state-is remarkably robust to the presence of interlayer exchange between the kagomé layers of its hexagonal crystal structure due to partial substitution at the interlayer sites or that the combination of chemically randomised magnetic interactions and geometric frustration results in a theoretically proposed disorder-induced $\mathrm{QSL}^{51}$. In either case, we conclude that $x \approx 0.5$ marks a critical threshold in the $\mathrm{Zn}$-barlowite series, above which dynamic magnetic moment correlations characterise the lowtemperature magnetic behaviour. We note that this is in agreement with a recent study which proposes that magnetic moments in single crystals of Zn-barlowite with $x=0.56$ remain dynamic to low-temperatures via bulk magnetometry measurements ${ }^{50}$.

In summary, we have demonstrated that the Zn-barlowite series offers a promising route to a new QSL phase in an $S=\frac{1}{2}$ kagomé antiferromagnet. By combining $\mu \mathrm{SR}$ experiment with DFT, we find that the electronic magnetic moments in the parent material of this series, barlowite, $\mathrm{Cu}_{4}(\mathrm{OH})_{6} \mathrm{FBr}$, are static below $T_{\mathrm{N}}=15 \mathrm{~K}$ and that $\mu \mathrm{SR}$ spectra can be quantitatively interpreted by the formation of both $\mu-\mathrm{F}$ and $\mu-\mathrm{OH}$ complexes. We note that the application of DFT methods may be especially important in the $\mu$ SR study of QSL candidates, as they allow us to assess not only the location of implanted muons but also the degree of distortion that they cause, 
thus providing confidence that the muon spin probes the intrinsic properties of the quantum disordered ground state ${ }^{52}$. In this regard, barlowite offers a valuable benchmark for DFT muon-site calculation and its application to other materials in which there is neither long-range magnetic order nor muon complex formation to give any indication as to where muons might implant. We find that incorporating as little as $x=0.16$ of $\mathrm{Zn}^{2+}$ into the crystal structure of barlowite leads to a suppression of its ordered, frozen fraction, while for $x>0.66$ the electronic magnetic moments remain dynamically fluctuating at all measurable temperatures. Whether the magnetic ground states of such partially substituted compositions of Zn-barlowite correspond to a QSL state-as proposed for the $x=1.0$ end-member ${ }^{21,29,30,50}$-awaits further investigation, for instance, by inelastic neutron scattering.

Also of note is that we find no evidence in our $\mu \mathrm{SR}$ measurements for the coexistence of long-range magnetic order and dynamic magnetic moment fluctuations in barlowite, which has been previously observed by other groups via NMR measurements ${ }^{53}$ and in the structurally related clinoatacamite via $\mu S R$ measurements ${ }^{33}$. Indeed, the reduced ordered moments obtained from the magnetic structure refinement of barlowite also indicate the presence of persistent dynamics in its ordered state ${ }^{23}$. However, an additional consideration here is the potential sample dependence on the magnetic properties of barlowite. Whilst the subtle structural differences depending on synthetic route are unlikely to have any significant effect on the muon stopping sites, some studies report several magnetic transitions in their samples synthesised by alternative routes to us which may eventually be insightful to explore using $\mu \mathrm{SR}^{20,22,24,50}$.

To conclude this present study, we finish by noting the striking similarity of the temperature dependence of the normalised muon spin relaxation rates, $\lambda_{\text {norm }}$, not only between members of the $\mathrm{Zn}$ barlowite series, but also with members of the related $\mathrm{Zn}$ paracamite series, and in particular, the QSL candidate herbertsmithite $^{16}$, as shown in Fig. 5b. The apparent equivalence of the dynamics of the magnetic moment fluctuations that drive both of these related families of $S=\frac{1}{2}$ kagomé antiferromagnets into the plateau state is perhaps indicative of a universality to the way in which such hydroxyl halides evolve towards a QSL regime upon chemical substitution. On the other hand, it could rather indicate the role of the interlayer $\mathrm{Cu}^{2+}$ moments present within the crystal structures of both systems, which has recently been proposed as an in situ probe of their QSL ground states via a Kondo-like interaction between the magnetic moments at the interlayer sites and the spinon excitations inherent to the possible QSL phases formed by the moments within their kagomé layers ${ }^{54}$. Further direct comparison of the evolution of magnetic moment correlations in the $\mathrm{Zn}_{x} \mathrm{Cu}_{4-x}(\mathrm{OH})_{6} \mathrm{FBr}$ and $\mathrm{Zn}_{x} \mathrm{Cu}_{4-x}(\mathrm{OH})_{6} \mathrm{Cl}_{2}$ series across complementary time and length scales by, for example, muon Knight shift, NMR spectroscopy or neutron spin-echo techniques, could, therefore, provide much-needed further experimental insight into the roles of interlayer coupling, cation occupancy disorder and local structure in the ground state selection of the $S=\frac{1}{2}$ kagomé antiferromagnet.

\section{METHODS}

Hydrothermal synthesis

Polycrystalline samples of barlowite, $\mathrm{Cu}_{4}(\mathrm{OH})_{6} \mathrm{FBr}$, were synthesised via a hydrothermal reaction as previously reported ${ }^{23}$. $Z n$-substituted samples, $\mathrm{Zn}_{x} \mathrm{Cu}_{4-x}(\mathrm{OH})_{6} \mathrm{FBr}$, were achieved through the addition of excess $\mathrm{ZnBr}_{2}$ whilst varying the amount of $\mathrm{CuBr}_{2}$ accordingly as detailed in Supplementary Table 1. For our fully $\mathrm{Zn}$-substituted sample, $x=0.99(1)$, the autoclave was heated at $1 \mathrm{~K} / \mathrm{min}$ to $483 \mathrm{~K}$, held for $24 \mathrm{~h}$ and cooled at a rate of $0.1 \mathrm{~K} / \mathrm{min}$ to room temperature. The resulting products, coloured turquoise to pale blue depending on the level of $\mathrm{Zn}$-substitution, were filtered and washed several times with distilled water and dried in air.
$\mathrm{XRD}$, inductively coupled plasma optical emission spectrometry (ICP-OES) and superconducting quantum interference device (SQUID) magnetometry

Sample purity was confirmed via powder X-ray diffraction (PXRD) using a Bruker d8 diffractometer in Debye-Scherrer geometry and utilising $\mathrm{Cu} \mathrm{K}_{\alpha 1}$ $(\lambda=0.154056 \AA)$ radiation. The $\mathrm{Cu}$ and $\mathrm{Zn}$ content was determined using ICP-OES on an Agilent 5110 SVDV ICP-OES. Samples were digested in dilute $\mathrm{HNO}_{3}$ and raw data are detailed in Supplementary Table 2. Time-of-flight PND measurements were performed on a sample containing $x=0.60(1)$ on the General Materials (GEM) diffractometer at the ISIS Facility of the Rutherford Appleton Laboratory ${ }^{55}$. The $1.4 \mathrm{~g}$ sample was loaded into a $6 \mathrm{~mm}$ vanadium can and measured at 2 and $200 \mathrm{~K}$. Magnetic susceptibility data were measured on a Quantum Design magnetic properties measurement system (MPMS) with a SQUID magnetometer. Samples were measured in VSM mode in an applied field of $1 \mathrm{~T}$ between 2 and $300 \mathrm{~K}$ in $2 \mathrm{~K}$ intervals.

\section{Muon spectroscopy measurements}

$\mu$ SR data were collected on the MuSR spectrometer at the ISIS Neutron and Muon Source ${ }^{56,57}$. Samples ranging from 60 to $500 \mathrm{mg}$ and $x=0.00$ to 0.99 (1) were packed into silver foil sachets with sizes ranging from 2.25 to $4 \mathrm{~cm}^{2}$, attached to a silver backing plate with vacuum grease and loaded into a ${ }^{4} \mathrm{He}$ cryostat. For $x=0.99(1)$ two additional silver sheets were placed in front of the sachet due to the small sample size of $60 \mathrm{mg}$. For $x=0.66(1)$ and $0.99(1)$, samples were additionally measured in a ${ }^{3} \mathrm{He} /{ }^{4} \mathrm{He}$ dilution fridge. Data were collected in zero-field (ZF), longitudinal-field (LF) and transverse-field (TF) geometries and analysed using the MANTID software ${ }^{58}$. A separate sample of $\mathrm{Cu}_{4}(\mathrm{OH})_{6} \mathrm{FBr}$ was measured on the General Purpose Surface-Muon (GPS) instrument of the Swiss Muon Source at Paul Scherrer Institut. The $90 \mathrm{mg}$ sample was contained in an aluminium foil packet, suspended in the muon beam using a silver fork-type holder and measured in VETO mode to reduce background signal.

\section{DFT calculations}

DFT muon-site calculations were carried out using the MuFinder software ${ }^{59}$ and the plane-wave-based code CASTEP $^{60}$ using the local density approximation. A supercell consisting of $1 \times 1 \times 2$ unit cells of the Pnma structural model of barlowite was used in order to minimise the effects of muon self-interaction resulting from the periodic boundary conditions. Muons, modelled by an ultrasoft hydrogen pseudopotential, were initialised in low-symmetry positions and the structure was allowed to relax (keeping the unit cell fixed) until the change in energy per ion was $<1 \times 10^{-5} \mathrm{eV}$. A cutoff energy of $544 \mathrm{eV}$ and a $1 \times 2 \times 1$ Monkhorst-Pack grid $^{61}$ were selected for $k$-point sampling.

\section{DATA AVAILABILITY}

Data supporting the findings within this study are available from the corresponding author upon request. Raw data sets from ISIS experiments can be accessed via links provided in refs. ${ }^{55-57}$.

\section{CODE AVAILABILITY}

The MuFinder program used to classify and analyse muon stopping sites can be downloaded, along with installation and usage instructions, via the link provided in ref. ${ }^{59}$.

Received: 25 May 2020; Accepted: 22 September 2020; Published online: 23 October 2020

\section{REFERENCES}

1. Greedan, J. E. Geometrically frustrated magnetic materials. J. Mater. Chem. 11, 37-53 (2001).

2. Broholm, C. et al. Quantum spin liquids. Science 367, eaay0668 (2020).

3. Knolle, J. \& Moessner, R. A field guide to spin liquids. Annu. Rev. Condens. Matter Phys. 10, 451-472 (2019).

4. Anderson, P. W. Resonating valence bonds: a new kind of insulator? Mat. Res. Bull. 8, 153-160 (1973).

5. Savary, L. \& Balents, L. Quantum spin liquids. Rep. Prog. Phys. 80, 016502 (2017).

6. Yan, S., Huse, D. A. \& White, S. R. Spin-liquid ground state of the $S=\frac{1}{2}$ kagome Heisenberg antiferromagnet. Science 332, 1173-1176 (2011). 
7. Han, T.-H. et al. Fractionalized excitations in the spin-liquid state of a kagomelattice antiferromagnet. Nature 492, 406-410 (2012).

8. Fu, M., Imai, T., Han, T.-H. \& Lee, Y. S. Evidence for a gapped spin-liquid ground state in a kagome Heisenberg antiferromagnet. Science 350, 655-658 (2015).

9. Khuntia, P. et al. Gapless ground state in the archetypal quantum kagome antiferromagnet $\mathrm{ZnCu}_{3}(\mathrm{OH})_{6} \mathrm{Cl}_{2}$. Nat. Phys. 16, 469-474 (2020).

10. Fåk, B. et al. Kapellasite: a kagome quantum spin liquid with competing interactions. Phys. Rev. Lett. 109, 037208 (2012).

11. Boldrin, D., Knight, K. \& Wills, A. S. Orbital frustration in the $S=\frac{1}{2}$ kagome magnet vesignieite kagome magnet vesignieite, $\mathrm{BaCu}_{3} \mathrm{~V}_{2} \mathrm{O}_{8}(\mathrm{OH})_{2}$. J. Mater. Chem. 4, 10315-10322 (2016).

12. Hiroi, Z., Ishikawa, H., Yoshida, H., Yamaura, J.-i. \& Okamoto, Y. Orbital transitions and frustrated magnetism in the kagome-type copper mineral volborthite. Inorg. Chem. 58, 11949-11960 (2019).

13. Shores, M. P., Nytko, E. A., Bartlett, B. M. \& Nocera, D. G. A structurally perfect $S=\frac{1}{2}$ kagomé antiferromagnet. J. Am. Chem. Soc. 127, 13462-13463 (2005).

14. Helton, J. S. et al. Spin dynamics of the spin-1/2 kagome lattice antiferromagnet $\mathrm{ZnCu}_{3}(\mathrm{OH})_{6} \mathrm{Cl}_{2}$. Phys. Rev. Lett. 98, 107204 (2007).

15. de Vries, M. A., Kamenev, K. V., Kockelmann, W. A., Sanchez-Benitez, J. \& Harrison, A. Magnetic ground state of an experimental $S=\frac{1}{2}$ kagome antiferromagnet. Phys. Rev. Lett. 100, 157205 (2008).

16. Mendels, P. et al. Quantum magnetism in the paratacamite family: towards an ideal kagomé lattice. Phys. Rev. Lett. 98, 077204 (2007).

17. Freedman, D. E. et al. Site specific X-ray anomalous dispersion of the geometrically frustrated kagomé magnet, herbertsmithite, $\mathrm{ZnCu}_{3}(\mathrm{OH})_{6} \mathrm{Cl}_{2}$. J. Am. Chem. Soc. 132, 16185-16190 (2010).

18. Nilsen, G. J., De Vries, M. A., Stewart, J. R., Harrison, A. \& Rønnow, H. M. Lowenergy spin dynamics of the $S=\frac{1}{2}$ kagome system herbertsmithite. J. Phys.: Condens. Matter 25, 106001 (2013)

19. Elliott, P., Cooper, M. A. \& Pring, A. Barlowite, $\mathrm{Cu}_{4} \mathrm{FBr}(\mathrm{OH})_{6}$, a new mineral isotructural with claringbullite: description and crystal structure. Mineral. Mag. 78, 1755-1762 (2014).

20. Han, T.-H., Singleton, J. \& Schlueter, J. A. Barlowite: a spin-1/2 antiferromagnet with a geometrically perfect kagome motif. Phys. Rev. Lett. 113, 227203 (2014).

21. Feng, Z. et al. Effect of $\mathrm{Zn}$ doping on the antiferromagnetism in kagome $\mathrm{Cu}_{4-x} \mathrm{Zn}_{x}(\mathrm{OH})_{6}$ FBr. Phys. Rev. B 98, 155127 (2018).

22. Pasco, $C$. M. et al. Single-crystal growth of $\mathrm{Cu}_{4}(\mathrm{OH})_{6} \mathrm{BrF}$ and universal behavior in quantum spin liquid candidates synthetic barlowite and herbertsmithite. Phys. Rev. Mater. 2, 044406 (2018).

23. Tustain, K., Nilsen, G. J., Ritter, C., da Silva, I. \& Clark, L. Nuclear and magnetic structures of the frustrated quantum antiferromagnet barlowite, $\mathrm{Cu}_{4}(\mathrm{OH})_{6} \mathrm{FBr}$. Phys. Rev. Mater. 2, 111405 (2018).

24. Smaha, R. W., He, W., Sheckelton, J. P., Wen, J. \& Lee, Y. S. Synthesis-dependent properties of barlowite and Zn-substituted barlowite. J. Solid State Chem. 268, 123-129 (2018)

25. Henderson, A. et al. Order-disorder transition in the $S=\frac{1}{2}$ antiferromagnets barlowite and claringbullite. Chem. Commun. 55, 11587-11590 (2019).

26. Han, T.-H., Isaacs, E. D., Schlueter, J. A. \& Singleton, J. Anisotropy: spin order and magnetization of single-crystalline $\mathrm{Cu}_{4}(\mathrm{OH})_{6} \mathrm{FBr}$ barlowite. Phys. Rev. B 93, 214416 (2016).

27. Liu, Z., Zou, X., Mei, J. W. \& Liu, F. Selectively doping barlowite for quantum spin liquid: a first-principles study. Phys. Rev. B 92, 220102(R) (2015).

28. Guterding, D., Valentí, R. \& Jeschke, H. O. Reduction of magnetic interlayer coupling in barlowite through isoelectronic substitution. Phys. Rev. B 94, 125136 (2016).

29. Feng, Z. et al. Gapped spin-1/2 spinon excitations in a new kagome quantum spin liquid compound $\mathrm{Cu}_{3} \mathrm{Zn}(\mathrm{OH})_{6} \mathrm{FBr}$. Chin. Phys. Lett. 34, 077502 (2017).

30. Wei, $Y$. et al. Evidence for a $Z_{2}$ topological ordered quantum spin liquid in a kagome-lattice antiferromagnet. Preprint at http://arxiv.org/abs/1710.02991 (2020).

31. Depenbrock, S., McCulloch, I. P. \& Schollwöck, U. Nature of the spin-liquid ground state of the $S=\frac{1}{2}$ Heisenberg model on the kagome lattice. Phys. Rev. Lett. 109, 067201 (2012)

32. Blundell, S. J. Spin-polarized muons in condensed matter physics. Contemp. Phys. 40, 175-192 (1999).

33. Zheng, X. G. et al. Coexistence of long-range order and spin fluctuation in geometrically frustrated clinoatacamite $\mathrm{Cu}_{2} \mathrm{ClOH}_{3}$. Phys. Rev. Lett. 95, 057201 (2005).

34. Kermarrec, E. et al. Spin-liquid ground state in the frustrated kagome antiferromagnet $\mathrm{MgCu}_{3}(\mathrm{OH})_{6} \mathrm{Cl}_{2}$. Phys. Rev. B 84, 100401 (2011).

35. Barthélemy, Q. et al. Local study of the insulating quantum kagome antiferromagnets $\mathrm{YCu}_{3}(\mathrm{OH})_{6} \mathrm{O}_{x} \mathrm{Cl}_{3-x}(x=0,1 / 3)$. Phys. Rev. Mater. 3, 074401 (2019).

36. Zorko, A. et al. Coexistence of magnetic order and persistent spin dynamics in a quantum kagome antiferromagnet with no intersite mixing. Phys. Rev. B 99, 214441 (2019).
37. Clark, L. et al. Gapless spin liquid ground state in the $S=\frac{1}{2}$ vanadium oxyfluoride kagome antiferromagnet $\left[\mathrm{NH}_{4}\right]_{2}\left[\mathrm{C}_{7} \mathrm{H}_{1} 4 \mathrm{~N}\right]\left[\mathrm{V}_{7} \mathrm{O}_{6} \mathrm{~F}_{1} 8\right]$. Phys. Rev. Lett. 110, 207208 (2013).

38. Foronda, F. R. et al. Anisotropic local modification of crystal field levels in Prbased pyrochlores: a muon-induced effect modeled using density functional theory. Phys. Rev. Lett. 114, 017602 (2015).

39. Möller, J. S. Playing quantum hide-and-seek with the muon: localizing muon stopping sites. Phys. Scr. 88, 068510 (2013).

40. Lord, J. S., Cottrell, S. P. \& Williams, W. G. Muon spin relaxation in strongly coupled systems. Phys. B Condens. Matter 289, 495-498 (2000).

41. Yaouanc, A. \& Dalmas de Réotier, P. Muon Spin Rotation, Relaxation, and Resonance (Oxford University Press, 2010).

42. Wei, Y. et al. Magnetic phase diagram of $\mathrm{Cu}_{4-x} \mathrm{Zn} \mathrm{n}_{x}(\mathrm{OH})_{6} \mathrm{FBr}$ studied by neutrondiffraction and $\mu \mathrm{SR}$ techniques. Preprint at http://arxiv.org/abs/2007.11816 (2020).

43. Slichter, C. P. Principles of Magnetic Resonance (Springer Verlag, 1978).

44. Gardner, J. S. et al. Cooperative paramagnetism in the geometrically frustrated pyrochlore antiferromagnet $\mathrm{Tb}_{2} \mathrm{Ti}_{2} \mathrm{O}_{7}$. Phys. Rev. Lett. 82, 1012-1015 (1999).

45. Li, Y. et al. Muon spin relaxation evidence for the $\mathrm{U}(1)$ quantum spin-liquid ground state in the triangular antiferromagnet $\mathrm{YbMgGaO}_{4}$. Phys. Rev. Lett. 117, 097201 (2016).

46. Mustonen, $O$. et al. Spin-liquid-like state in a spin-1/2 square-lattice antiferromagnet perovskite induced by $d^{10}-d^{0}$ cation mixing. Nat. Commun. 9, 1085 (2018).

47. Kenney, E. M. et al. Coexistence of static and dynamic magnetism in the Kitaev spin liquid material $\mathrm{Cu}_{2} \mathrm{IrO}_{3}$. Phys. Rev. B 100, 094418 (2019).

48. Dunsiger, S. R. et al. Muon spin relaxation investigation of the spin dynamics of geometrically frustrated antiferromagnets $\mathrm{Y}_{2} \mathrm{Mo}_{2} \mathrm{O}_{7}$ and $\mathrm{Tb}_{2} \mathrm{Mo}_{2} \mathrm{O}_{7}$. Phys. Rev. $B$ 54, 9019-9022 (1996).

49. Gomilšek, M. et al. $\mu \mathrm{SR}$ insight into the impurity problem in quantum kagome antiferromagnets. Phys. Rev. B 94, 024438 (2016).

50. Smaha, R. W. et al. Materializing rival ground states in the barlowite family of kagome magnets: quantum spin liquid, spin ordered, and valence bond crystal states. npj Quant. Mater. 5, 23 (2020).

51. Kawamura, H., Watanabe, K. \& Shimokawa, T. Quantum spin-liquid behavior in the spin- $\frac{1}{2}$ random-bond Heisenberg antiferromagnet on the kagome latticeJ. Phys. Soc. Jpn. 83, 103704 (2014).

52. Lancaster, T. et al. Quantum magnetism in molecular spin ladders probed with muon-spin spectroscopy. N. J. Phys. 20, 103002 (2018).

53. Ranjith, K. M. et al. Magnetic resonance as a local probe for kagome magnetism in barlowite $\mathrm{Cu}_{4}(\mathrm{OH})_{6} \mathrm{FBr}$. Sci. Rep. 8, 10851 (2018).

54. Gomilšek, M. et al. Kondo screening in a charge-insulating spinon metal. Nat. Phys. 15, 754-758 (2019).

55. Tustain, K. et al. STFC ISIS Facility, https://doi.org/10.5286/ISIS.E.95671117 (2018). 56. Tustain, K. et al. STFC ISIS Facility, https://doi.org/10.5286/ISIS.E.RB1720027 (2018).

57. Tustain, K. et al. STFC ISIS Facility, https://doi.org/10.5286/ISIS.E.RB1910387 (2019).

58. Arnold, O. et al. Mantid-Data analysis and visualization package for neutron scattering and $\mu$ SR experiments. Nucl. Instrum. Methods Phys. Res. 764, 156-166 (2014).

59. Huddart, B. M. https://gitlab.com/BenHuddart/mufinder/ (2020).

60. Clark, S. J. et al. First principles methods using CASTEP. Z. Kristallogr. 220, 567-570 (2005)

61. Monkhorst, H. J. \& Pack, J. D. Special points for Brillouin-zone integrations. Phys. Rev. B 13, 5188 (1976)

\section{ACKNOWLEDGEMENTS}

L.C. and K.T. acknowledge the University of Liverpool for financial support and a studentship to K.T. B.M.H. thanks the STFC for the provision of a studentship. T.-H.H. acknowledges the support of the Grainger Fellowship provided by the University of Chicago. Work at the ISIS Neutron and Muon Source was supported by the STFC. Part of this work is supported by the EPSRC grant no EP/N024028/1. F.B. acknowledges support from the French Agence Nationale de la Recherche under Grant No. ANR-18CE30-0022 LINK and from the Universite Paris-Sud (MRM MAGMAG grant). The authors are grateful to S. Moss and the University of Liverpool Analytical Services for performing ICP-OES measurements, $\mathrm{H}$. Niu and $\mathrm{B}$. Le Pennec for assistance with susceptibility measurements, as well as I. da Silva for assistance on GEM. We acknowledge computing resources provided by Durham Hamilton HPC.

\section{AUTHOR CONTRIBUTIONS}

K.T., B.W.-O' B. and T.-H.H. prepared the samples. K.T., F.B., P.J.B. and L.C. performed the MuSR experiments and K.T. analysed the data. F.B. and H.L. performed the GPS 
experiments and F.B. analysed the data. T.L. and B.M.H. performed the DFT calculations. K.T. performed supplementary experiments (magnetic susceptibility and PND) and analysed the data. L.C. conceived of and supervised the study. L.C. and K.T. wrote the manuscript with input from all of the authors.

\section{COMPETING INTERESTS}

The authors declare no competing interests.

\section{ADDITIONAL INFORMATION}

Supplementary information is available for this paper at https://doi.org/10.1038/ s41535-020-00276-4.

Correspondence and requests for materials should be addressed to L.C.

Reprints and permission information is available at http://www.nature.com/ reprints
Publisher's note Springer Nature remains neutral with regard to jurisdictional claims in published maps and institutional affiliations.

Open Access This article is licensed under a Creative Commons cc) Attribution 4.0 International License, which permits use, sharing, adaptation, distribution and reproduction in any medium or format, as long as you give appropriate credit to the original author(s) and the source, provide a link to the Creative Commons license, and indicate if changes were made. The images or other third party material in this article are included in the article's Creative Commons license, unless indicated otherwise in a credit line to the material. If material is not included in the article's Creative Commons license and your intended use is not permitted by statutory regulation or exceeds the permitted use, you will need to obtain permission directly from the copyright holder. To view a copy of this license, visit http://creativecommons. org/licenses/by/4.0/.

(c) The Author(s) 2020 